\title{
Topographical Central Island-Like Pattern After 24 Hrs of Continuous Intraocular Pressure Monitoring with a Contact Lens Sensor
}

This article was published in the following Dove Press journal:

International Medical Case Reports Journal

\section{Hiroshi Toshida (D)}

Department of Ophthalmology, Juntendo University Shizuoka Hospital, Izunokuni, Shizuoka, Japan
Correspondence: Hiroshi Toshida Department of Ophthalmology, Juntendo University Shizuoka Hospital, Izunokuni, Shizuoka 4I0-2295, Japan

Tel +8I 559483 III

Fax +8I 55948335 I

Email toshida@juntendo.ac.jp

\begin{abstract}
With development of the contact lens sensor (CLS), it has become possible to monitor the intraocular pressure (IOP) for 24 hrs continuously. Wearing of CLS often brings blurred vision with transient aggravation of myopia and changes in corneal shape. The author, a 51-year-old man with myopic astigmatism, wore a CLS for $24 \mathrm{hrs}$ on the right eye, and the fellow eye served as a contra-lateral control eye. After wearing, his corrected visual acuity on the right eye decreased from $20 / 16$ to $20 / 25$ with blurred vision, and subjective spherical power and cylindrical power aggravated. Topographical analysis revealed that the instantaneous power increased on the central cornea but decreased on the mid-peripheral cornea. Differential instantaneous map of pre- and post-wearing CLS showed a specific pattern similar to the central island pattern, which is known as the results of steeper fitting of the orthokeratology lens. A surface imprint was observed on the bulbar conjunctiva, corresponding to the edge of the contact lens. These findings seemed due to orthokeratological effects by the steeper fitting of CLS. All of them resolved within $24 \mathrm{hrs}$ after the removal of the CLS.
\end{abstract}

Keywords: contact lens sensor, CLS, triggerfish, topography, orthokeratological effects, central island

\section{Introduction}

It is well known that the intraocular pressure (IOP) varies throughout the day not only glaucoma patients but normal subjects. ${ }^{1,2}$ In patients with glaucoma, the IOP is usually measured once at a clinic, so the pattern of IOP values during daily life is unknown. Diurnal variation of the IOP is observed in people with healthy eyes as well as patients with glaucoma, and the pattern of such diurnal variation differs widely between individuals and according to body posture. ${ }^{1-4}$ To assess the diurnal variation of the IOP, it used to be necessary for a patient to be admitted to a hospital and undergo repeated IOP measurement over $24 \mathrm{hrs}$. This method of evaluation placed a heavy burden on both patients and ophthalmologists.

After the SENSIMED Triggerfish ${ }^{\circledR}$ contact lens sensor (CLS [Sensimed AG, Lausanne, Switzerland]) was developed in 2009, it became possible to measure the IOP continuously over $24 \mathrm{hrs}$ by wearing a CLS. ${ }^{5,6}$ Therefore, availability of the CLS has reduced the burden of investigating the IOP for both patients and ophthalmologists. The CLS is placed on the eye to be tested and monitors the changes of corneal curvature induced by changes of the IOP, while transmitting measurements 
to a "Triggerfish ${ }^{\circledR}$ " receiver that records the data. ${ }^{7}$ This system can be used to detect the peaks of IOP.

Most of the previous studies were performed in participants with spherical power between -5 and 3 diopters (D) and cylindrical power $2 \mathrm{D}$ or less, ${ }^{8-13}$ awareness of blurred vision is one of the main frequent adverse events with CLS. ${ }^{8,10,14-16}$ Although the CLS is available in three curvature radius sizes: steep, medium, and flat, ${ }^{8}$ curvature radius size of CLS is selected based on the measured corneal curvature radius of the patient's eyes. As the fitting of CLS generally tight, wearing of a CLS often brings transient aggravation of myopia with changes in corneal shape and refractive power.

In the present study, a participant who had myopic astigmatism showed a specific pattern similar to the central island pattern ${ }^{17}$ caused by the steeper fitting of orthokeratology lens in the differential instantaneous map of pre- and post-wearing lenses. This topographical pattern showing a perfectly centered area of central corneal steepening, surrounded by a "moat" of marked flattening seemed due to orthokeratological effects by tight fitting of $\mathrm{CLS}^{8}$

A

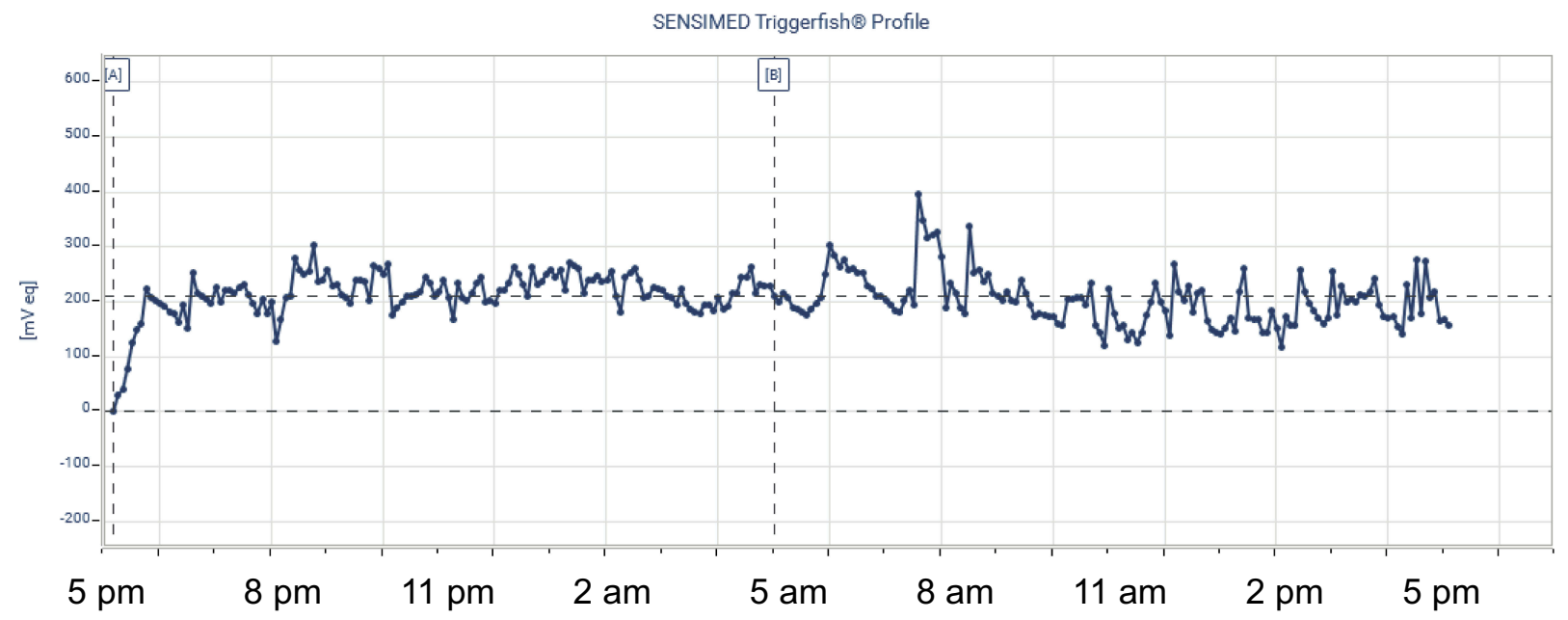

(hours)

B

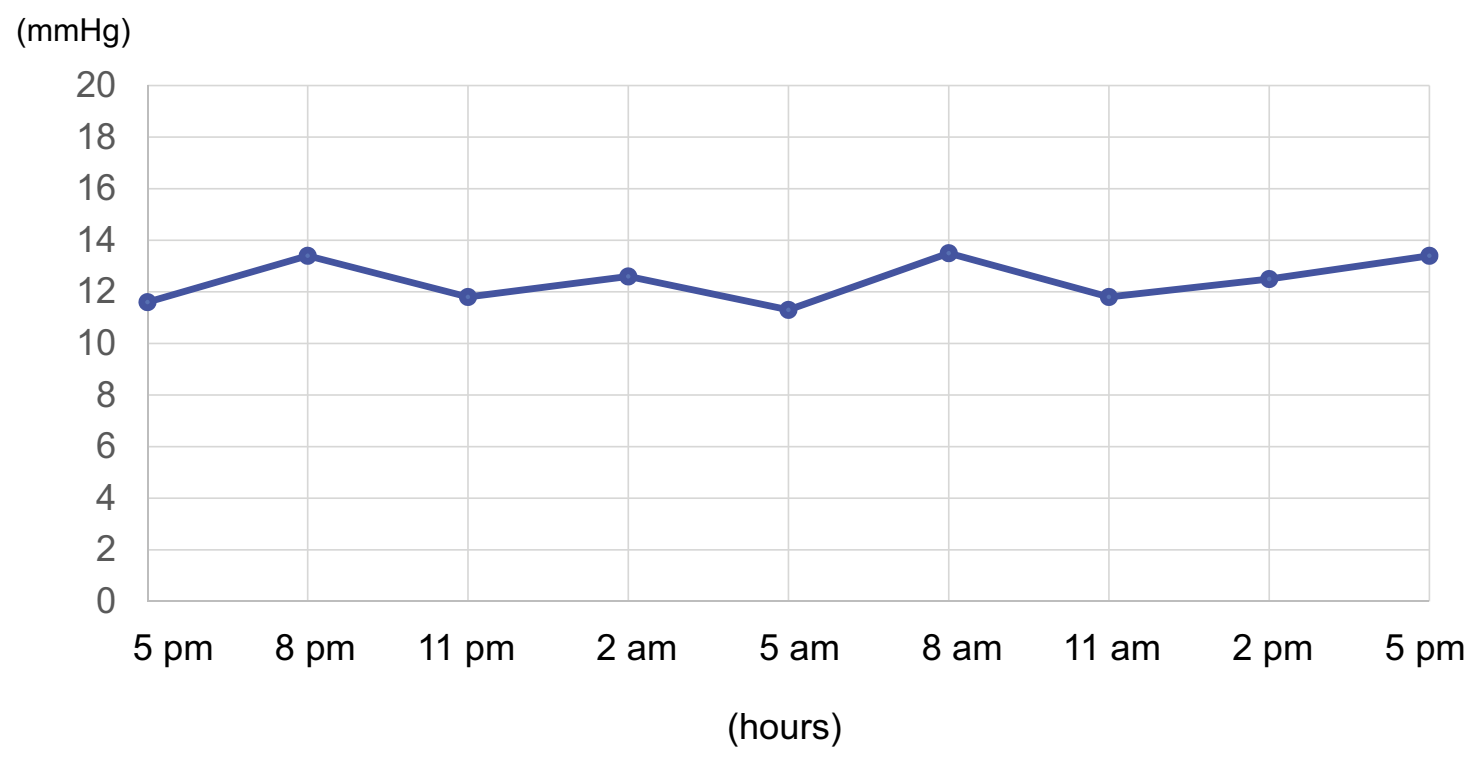

Figure I Results of IOP.

Notes: (A) IOP measured by $24 \mathrm{hr}$ continuous monitoring by the CLS in the right eye. (B) IOP measured by a noncontact tonometer every 3 hrs in the left eye. The IOP did not change markedly in both eyes during $24 \mathrm{hr}$ period. 


\section{Materials and Methods}

The author, a 51-year-old healthy man who had myopic astigmatism without glaucoma (author) participated in the present study. On the day before wearing the CLS, after checking the eyes, the objective refraction value measured by automated refraction and keratometry (ARK-1a, Nidek, Gamagori, Japan), subjective refraction value and visual acuity were examined. The IOP was measured by noncontact tonometer (Icare ${ }^{\mathbb{R}}$ PRO tonometer, Icare Finland Oy, Vantaa, Finland), and the shape of the cornea and corneal thickness were determined by anterior-segment optical coherence tomography (AS-OCT, SS-1000 CASIA, Tomey, Nagoya, Japan) as the previous study. ${ }^{17}$ The average power and corneal thickness in paracentral, mid-peripheral and peripheral cornea shown on the AC-OCT map were also calculated by each 8 measurement points on the concentric circles with diameter of 3,6 and 9mm, respectively.

A CLS was placed on the right eye of the subject at 5 p.m. to continuously measure the IOP for $24 \mathrm{hrs}$. In addition, the IOP of the left eye was measured every $3 \mathrm{hrs}$ from 5 p.m. by noncontact tonometer. After 24 hrs (ie, at 5 p.m. on the next day), the CLS was removed from the right eye, and the same examinations performed before wearing the CLS were repeated. In addition, fluorescein staining and the rose Bengal staining of the cornea and conjunctiva were performed. All of these examinations were done again at $48 \mathrm{hrs}$ after the start of CLS use.

Wilcoxon $t$-test was used to compare the results of average power and corneal thickness measured by ASOCT before and after CLS wearing and significance was set at $\mathrm{p}<0.05$.

\section{Results}

As the mean keratometry was $7.79 \mathrm{~mm}$, a CLS with a medium curvature of $8.7 \mathrm{~mm}$ was chosen by the manufacture's manual. The results of IOP obtained by $24 \mathrm{hr}$ continuous monitoring using the CLS in the right eye in Figure 1A and IOP by a noncontact tonometer every $3 \mathrm{hrs}$ in the left eye are shown in Figure 1B. Before wearing the CLS, the IOP measured by a noncontact tonometer was $13.0 \mathrm{mmHg}$ for the right eye and $11.6 \mathrm{mmHg}$ for the left eye (Table 1). During the $24 \mathrm{hr}$ period, the IOP did not change markedly in either eye, and the pattern of IOP variation corresponded to the "no significant acrophase" pattern described in past reports. ${ }^{9}$ Immediately after removal of the CLS from the right eye, the IOP measured by a noncontact tonometer was $13.6 \mathrm{mmHg}$ for the right eye and $13.4 \mathrm{mmHg}$ for the left eye.

His corrected visual acuity on the right eye decreased from $24 / 20$ to $16 / 20$ after wearing a CLS. With regard to subjective refraction, the spherical power was increased from $-1.75 \mathrm{D}$ to $-3.75 \mathrm{D}$, and cylindrical power slightly increased from $-1.75 \mathrm{D}$ to $-2.00 \mathrm{D}$ after wearing the CLS for $24 \mathrm{hrs}$ (Table 1). The spherical equivalent refractive error (D) was also increased from $-2.63 \mathrm{D}$ to $-4.75 \mathrm{D}$. Figure 2 shows the data from the topographic map of the axial power and pachymetry before wearing CLS and Figure 3 just after removal of CLS at $24 \mathrm{hrs}$. There were obvious changes in the anterior and the real power. Instantaneous power also increased on the corneal center

Table I Results of Ophthalmological Examinations Before and After Wearing CLS

\begin{tabular}{|c|c|c|c|}
\hline & Before & $24 \mathrm{Hr}$ After & p value \\
\hline \multicolumn{4}{|l|}{ Intraocular Pressure $(\mathrm{mmHg})$} \\
\hline Experimental side (right eye) & 13.0 & 13.6 & \\
\hline $\begin{array}{l}\text { Contra-lateral control side } \\
\text { (left eye) }\end{array}$ & 11.6 & 13.4 & \\
\hline Uncorrected visual acuity & $2 / 20$ & $2 / 20$ & \\
\hline Corrected visual acuity & $24 / 20$ & $16 / 20$ & \\
\hline \multicolumn{4}{|l|}{ Keratometry } \\
\hline KM (D) & 43.25 & 38.25 & \\
\hline $\mathrm{K} \max (\mathrm{D})$ & 45.00 & 38.75 & \\
\hline Kmin (D) & 41.75 & 37.50 & \\
\hline cyl (D) & -3.25 & -2.75 & \\
\hline Axis & $178^{\circ}$ & $11^{\circ}$ & \\
\hline \multicolumn{4}{|l|}{ Subjective Refraction } \\
\hline Spherical power (D) & -1.75 & -3.75 & \\
\hline Cylinder power (D) & -1.75 & -2.00 & \\
\hline Axis & $180^{\circ}$ & $180^{\circ}$ & \\
\hline $\begin{array}{l}\text { Spherical equivalent } \\
\text { refractive error }(D)\end{array}$ & -2.63 & -4.75 & \\
\hline \multicolumn{4}{|c|}{ Instantaneous Power (keratometric) } \\
\hline Corneal center (D) & 42.00 & 45.00 & \\
\hline Paracentral cornea (D) & $42.55 \pm 1.34$ & $40.53 \pm 4.46$ & \\
\hline Midperipheral cornea (D) & $41.50 \pm 1.97$ & $33.94 \pm 4.04$ & $p=0.0117 *$ \\
\hline Peripheral cornea (D) & $36.35 \pm 3.20$ & $40.10 \pm 7.18$ & \\
\hline \multicolumn{4}{|l|}{ Corneal Thickness } \\
\hline Corneal center $(\mu \mathrm{m})$ & 515 & 528 & \\
\hline Paracentral cornea $(\mu \mathrm{m})$ & $528.6 \pm 15.1$ & $533.8 \pm 10.4$ & \\
\hline Midperipheral cornea $(\mu \mathrm{m})$ & $554.8 \pm 25.3$ & $559.5 \pm 32.8$ & \\
\hline Peripheral cornea $(\mu \mathrm{m})$ & $591.8 \pm 35.4$ & $620.8 \pm 48.5$ & $p=0.0117^{*}$ \\
\hline
\end{tabular}

Notes: Results are partially expressed as the mean, SD. *Wilcoxon $t$-test. Abbreviations: MK, mean keratometry; D, diopters; Kmax, maximum keratometry; Kmin, minimum keratometry. 


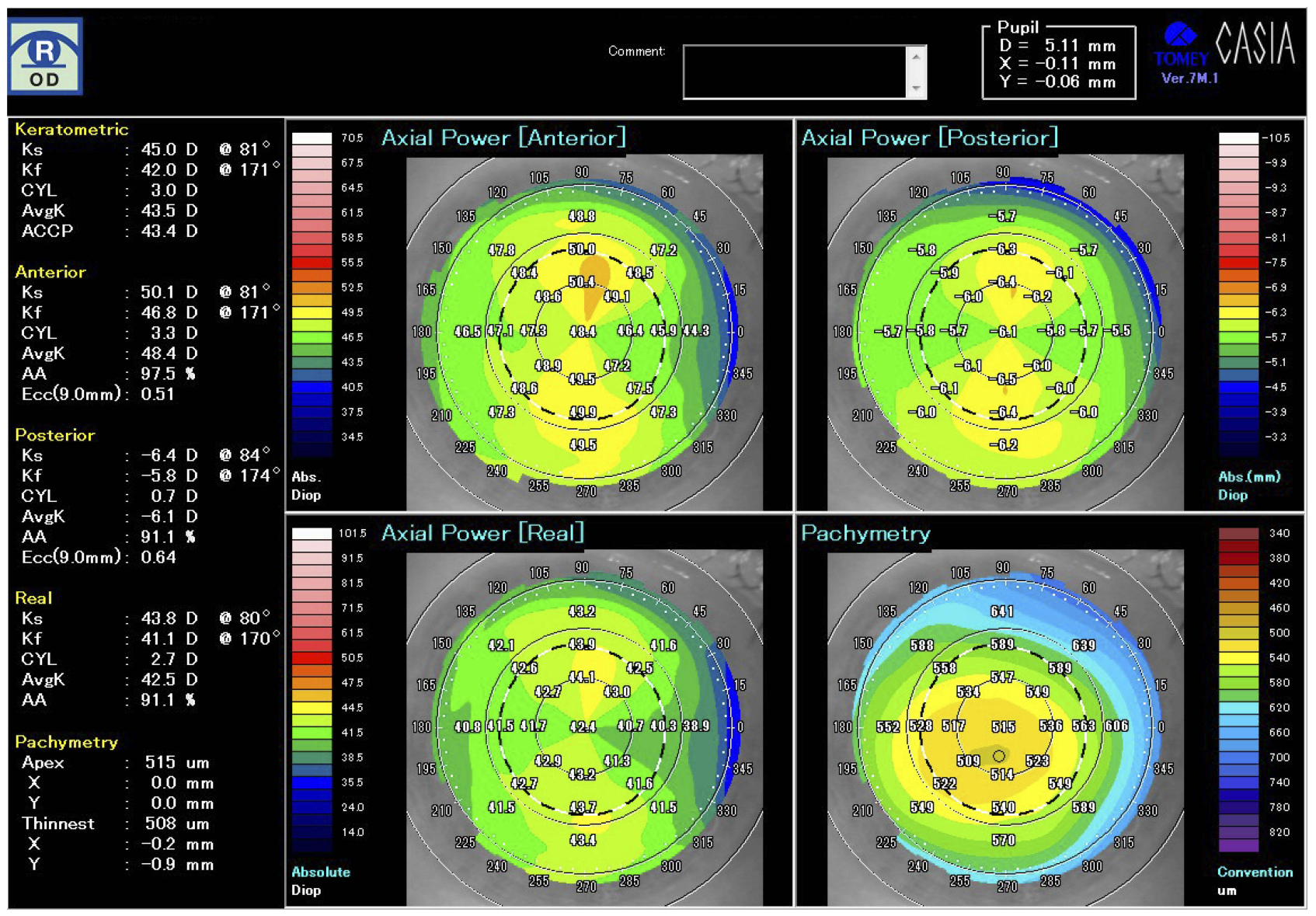

Figure 2 The topographic map of the axial power and pachymetry before placement of CLS.

and decreased on the mid-peripheral cornea after wearing the CLS compared with baseline (Figure 4). The differential map (Figure 4) of the instantaneous power measured at pre-and post-wearing CLS showed a specific pattern similar to the central island pattern caused by steeper orthokeratology lens fitting. Thus, the corneal curvature became steeper and the cylindrical power was increased, so astigmatism was aggravated. While wearing the CLS and after its removal, the subject noted blurring of his vision. The central corneal thickness increased from 515 $\mu \mathrm{m}$ before wearing the CLS to $528 \mu \mathrm{m}$ after $24 \mathrm{hrs}$, and corneal thickness averaged by 8 points in the peripheral cornea also increased from $591.8 \pm 35.4 \mu \mathrm{m}$ to $620.8 \pm$ $48.5 \mu \mathrm{m}$ statistically $(\mathrm{p}=0.0117$, wilcoxson $t$-test $)$. The power on the mid-peripheral cornea averaged by 8 points decreased statistically ( $\mathrm{p}=0.0117$, wilcoxson $t$-test). At 24 hrs after removing the CLS (ie, $48 \mathrm{hrs}$ after the start of this experiment), most of the measured values returned to baseline as following; the visual acuity returned to 24/ 20 , the spherical power to $-1.75 \mathrm{D}$, spherical equivalent refractive error (D) to $-2.75 \mathrm{D}$. Instantaneous power of keratometric in the mid-peripheral cornea to $41.59 \pm$ $2.58 \mathrm{D}$ and corneal thickness in the peripheral cornea to $598.8 \pm 32.4 \mu \mathrm{m}$.

The ocular findings at just starting CLS wear (A) and at $1 \mathrm{hr}$ after CLS wear (B) is shown in Figure 5. The CLS is fixed with slight inferior displacement and mild bulbar conjunctival hyperemia can be observed. The ocular findings immediately after removal of the CLS following $24 \mathrm{hr}$ continuous measurement of the IOP are shown in Figure 6A. The bulbar conjunctiva showed obvious hyperemia. The superficial punctate keratitis stained by fluorescein was positive in the superior temporal mid-peripheral segment of the cornea (arrowhead) and inferior segment (arrow) (Figure 6B). Rose Bengal staining revealed a surface imprint on the bulbar conjunctiva, corresponding to the edge of the CLS (Figure 6C). Both while wearing the CLS and after removal of the CLS, the subject noted intermittent itching of the eye. After removal of the CLS, the subject instilled $0.1 \%$ hyaluronic acid eye drops 3 times during the first 24 hrs. Both the objective findings and the subjective 


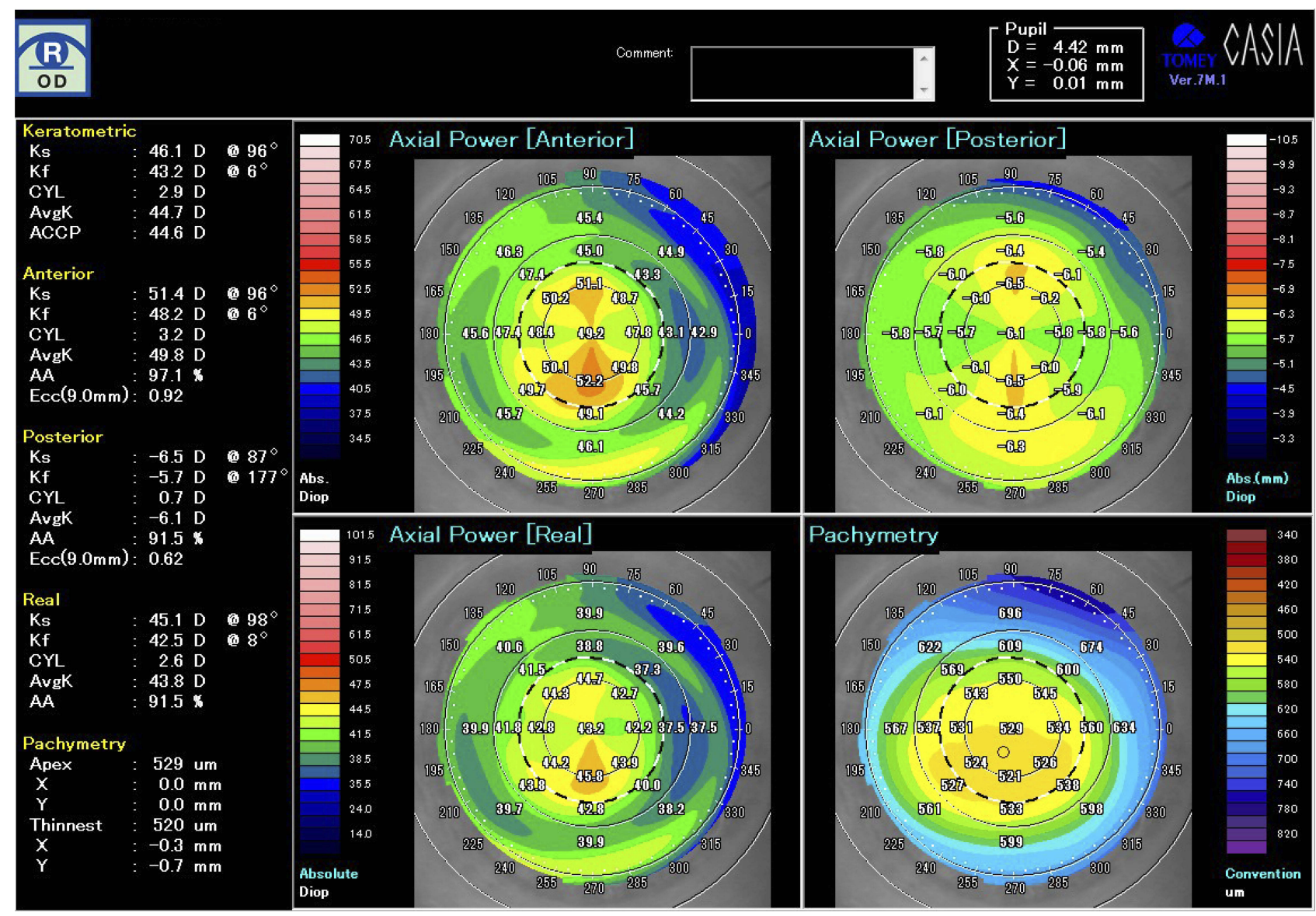

Figure 3 The topographic map of the axial power and pachymetry just after removal of CLS after 24 hr continuous monitoring of IOP.

symptoms resolved within $24 \mathrm{hrs}$ after removal of the contact lens, ie, within $48 \mathrm{hrs}$ after the start of this experiment.

\section{Discussion}

The first report on the $24 \mathrm{hr}$ continuous measurement of the IOP using the CLS was published in $2011 .{ }^{6}$ Since then, measurements of the IOP obtained using the CLS in patients with glaucoma, healthy subjects, and patients with sleep apnea syndrome have been reported. ${ }^{7-16,18}$ However, there have been relatively a few reports about the influences of the CLS on the cornea. ${ }^{13,19-21}$ After wearing the CLS, the most common corneal/conjunctival finding is reported to be bulbar conjunctival hyperemia, followed by superficial punctate keratopathy and corneal epithelial erosion. ${ }^{6,8,10,14-16,22}$ In contrast, there have been few reports about the proper fitting of the CLS. In the present study, bulbar conjunctival hyperemia and superficial punctate keratopathy were noted after wearing the CLS, but these changes resolved within $24 \mathrm{hrs}$. It was thought that these changes occurred because the curvature of the CLS was too steep for the subject's eye. In fact, the CLS stuck to the eye, so, the instillation of an anesthetic eye drop was required when the CLS was removed and forceps were also needed. After wearing the CLS for 24 hrs, visual acuity decreased with blurred vision, and aggravation of myopia and astigmatism was noted transiently, indicating that the corneal curvature had become steeper on the corneal center. It seemed to be due to steeper fitting of the CLS, and transient myopization is in agreement with the previous reports. ${ }^{8,10,13,19,21}$ Furthermore, topographic analysis in the present study revealed that the instantaneous power increased on the central cornea but decreased on the mid-peripheral cornea. Differential map of the instantaneous power comparing pre- and post-wearing of CLS showed a specific pattern was similar to the central island pattern caused by the steeper fitting of orthokeratology lenses. ${ }^{8,17}$ So, it is thought that both are caused by an orthokeratological effect with steeper lens fitting.

According to the manufacturer of the CLS, even if the curvature of the lens selected by measurement of the patient's eye is too steep, the selected lens cannot be changed for 


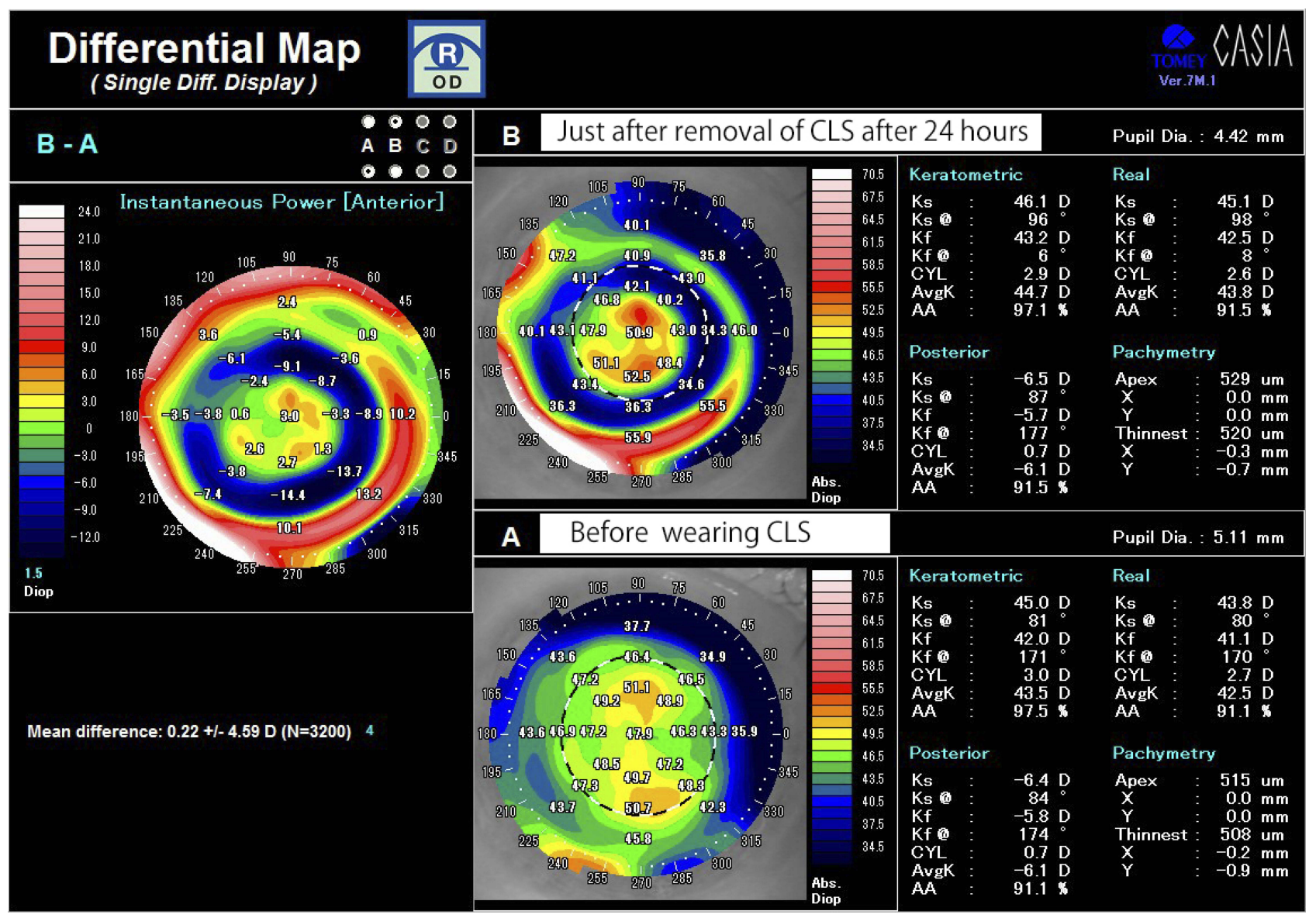

Figure 4 The differential map of the instantaneous power measured at pre-and post-wearing CLS.

Note: It showed a specific pattern shaped to resemble central island pattern caused by steeper orthokeratology lens fitting.

A

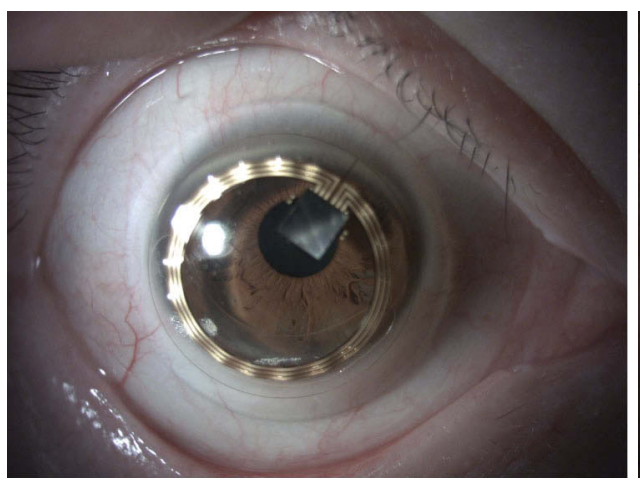

B

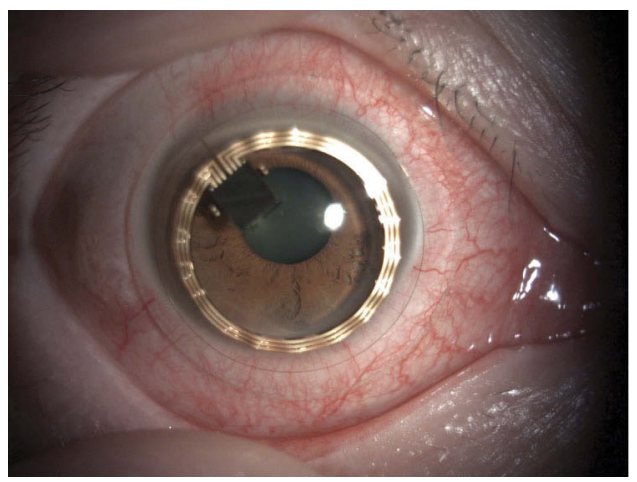

Figure 5 Ocular findings of the anterior segment at just placed the CLS and at I hr after starting.

Notes: (A) CLS on the right eye just after placed. There was no hyperemia. (B) The CLS is fixed with the bulbar conjunctiva and mild bulbar conjunctival hyperemia was shown.

another CLS with a different curvature. There are three curvature radius sizes for the CLS, which are termed steep, medium, and flat. Unlike the method for fitting a conventional soft contact lens (SCL), a CLS with a steep, medium, or flat curvature is selected by measurement of the patient's corneal curvature radius. The manufacturer's manual for the CLS specifies that a lens with a steep curvature of $8.4 \mathrm{~mm}$, a lens with a medium curvature of $8.7 \mathrm{~mm}$, and a lens with a flat curvature of $9.0 \mathrm{~mm}$ should be selected for eyes with a corneal curvature radius of $7.53 \mathrm{~mm}$ or less, 
A

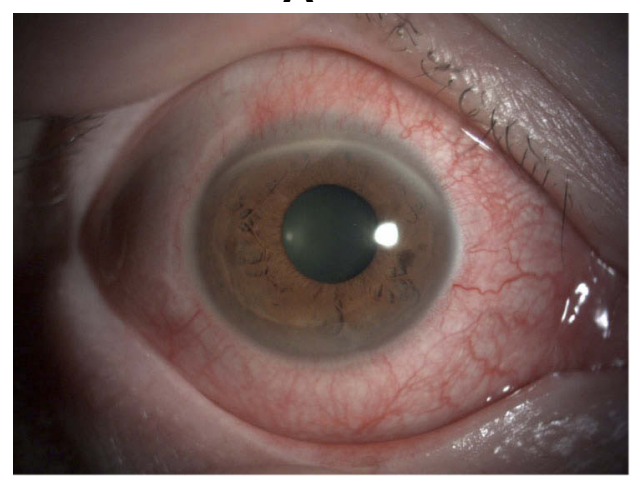

B

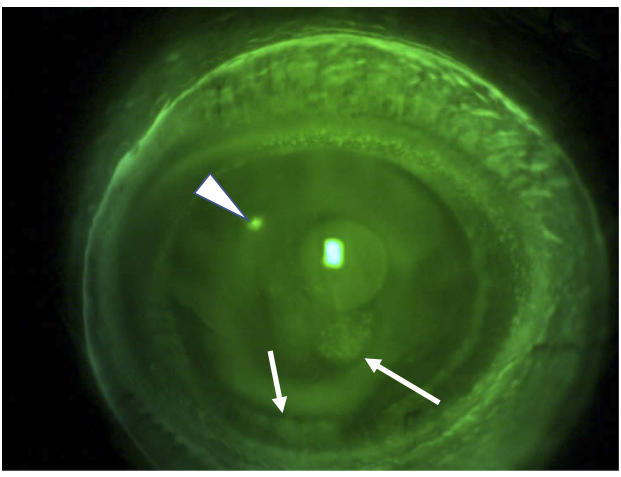

C

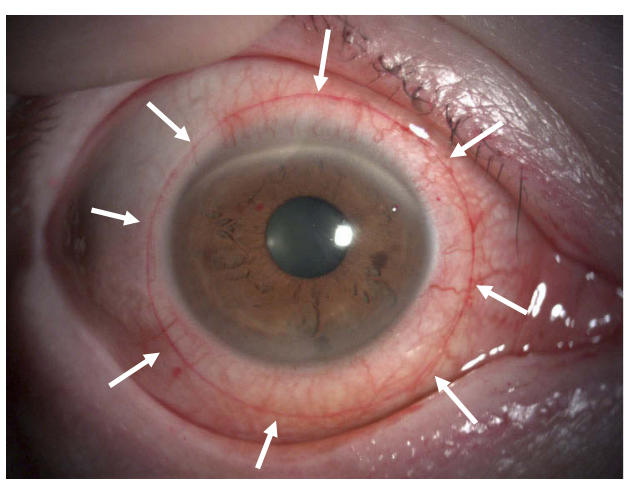

Figure 6 Ocular findings just after removal of the CLS following $24 \mathrm{hr}$ continuous measurement of the IOP.

Notes: (A) The bulbar conjunctiva showed obvious hyperemia. (B) Fluorescein staining. The superficial punctate keratitis was shown in superior temporal mid-peripheral (arrowhead) and inferior segment (arrow). (C) Rose Bengal staining. The surface imprint on the bulbar conjunctiva, corresponding to the edge of the CLS was observed (arrow).

7.54-8.44 $\mathrm{mm}$, and $8.45 \mathrm{~mm}$ or more, respectively. With regard to ordinary SCLs, if the fit of the selected lens is too tight, the prescription is generally changed for a new SCL with a larger curvature. With the Triggerfish system, however, there is no opportunity for correction of the CLS selected by a physician. Furthermore, it has been reported that the shape of the cornea is different between Asians and Caucasians. ${ }^{23}$ Therefore, it might be required that a separate CLS instruction manual for Asians.

\section{Conclusion}

After the removal of the CLS, the subject of the present study noted transient blurred vision with aggravation of myopia and astigmatism. Furthermore, formation of central island-like pattern was shown by topographical analysis, due to orthokeratological effects by tight fitting of CLS. These findings resolved within $24 \mathrm{hrs}$ after the removal of the CLS.

\section{Ethics}

The institutional review board at Juntendo University Shizuoka Hospital did not require the author to obtain ethics approval and patient consent, because the participant was the author.

\section{Acknowledgment}

This study was supported in part by the Ministry of Education, Culture, Sports, Science and Technology (MEXT)-Supported Program from the Strategic Research Foundation at Private Universities, 2015 to 2019.

\section{Disclosure}

Dr Hiroshi Toshida reports grants from the Ministry of Education, Culture, Sports, Science and Technology, during the conduct of the study and outside the submitted work. The author reports no other conflicts of interest in this work.

\section{References}

1. Liu JH, Kripke DF, Hoffman RE, et al. Nocturnal elevation of intraocular pressure in young adults. Invest Ophthalmol Vis Sci. 1998;39 (13):2707-2712.

2. Liu JH, Zhang X, Kripke DF, Weinreb RN. Twenty-four-hour intraocular pressure pattern associated with early glaucomatous changes. Invest Ophthalmol Vis Sci. 2003;44(4):1586-1590. doi:10.1167/ iovs.02-0666 
3. Hara T, Hara T, Tsuru T. Increase of peak intraocular pressure during sleep in reproduced diurnal changes by posture. Arch Ophthalmol. 2006;124(2):165-168. doi:10.1001/archopht.124.2.165

4. Kiuchi T, Motoyama Y, Oshika T. Relationship of progression of visual field damage to postural changes in intraocular pressure in patients with normal-tension glaucoma. Ophthalmology. 2006;113 (12):2150-2155. doi:10.1016/j.ophtha.2006.06.014

5. Leonardi M, Pitchon EM, Bertsch A, Renaud P, Mermoud A. Wireless contact lens sensor for intraocular pressure monitoring: assessment on enucleated pig eyes. Acta Ophthalmol. 2009;87 (4):433-437. doi:10.1111/j.1755-3768.2008.01404.x

6. Mansouri K, Shaarawy T. Continuous intraocular pressure monitoring with a wireless ocular telemetry sensor: initial clinical experience in patients with open angle glaucoma. Br J Ophthalmol. 2011;95 (5):627-629. doi:10.1136/bjo.2010.192922

7. Mansouri K, Weinreb R. Continuous 24-hour intraocular pressure monitoring for glaucoma-time for a paradigm change. Swiss Med Wkly. 2012;142:w13545. eCollection 2012. doi:10.4414/ smw.2012.13545

8. Mansouri K, Medeiros FA, Tafreshi A, Weinreb RN. Continuous 24-hour monitoring of intraocular pressure patterns with a contact lens sensor: safety, tolerability, and reproducibility in patients with glaucoma. Arch Ophthalmol. 2012;130(12):1534-1539.

9. Mansouri K, Liu JH, Weinreb RN, Tafreshi A, Medeiros FA. Analysis of continuous 24-hour intraocular pressure patterns in glaucoma. Invest Ophthalmol Vis Sci. 2012;53(13):8050-8056.

10. Lorenz K, Korb C, Herzog N, et al. Tolerability of 24-hour intraocular pressure monitoring of a pressure-sensitive contact lens. J Glaucoma. 2013;22(4):311-316. doi:10.1097/IJG.0b013e318241b874

11. Mottet B, Aptel F, Romanet JP, Hubanova R, Pépin JL, Chiquet C. 24hour intraocular pressure rhythm in young healthy subjects evaluated with continuous monitoring using a contact lens sensor. JAMA Ophthalmol. 2013;131(12):1507-1516. doi:10.1001/jamaophthalmol.2013.5297

12. Aptel F, Tamisier R, Pépin JL, et al. Hourly awakening vs continuous contact lens sensor measurements of 24-hour intraocular pressure: effect on sleep macrostructure and intraocular pressure rhythm. JAMA Ophthalmol. 2014;132(10):1232-1238. doi:10.1001/jamaophthalmol. 2014.1761

13. Hubanova R, Aptel F, Chiquet C, Mottet B, Romanet JP. Effect of overnight wear of the Triggerfish $\left({ }^{\circledR}\right)$ sensor on corneal thickness measured by Visante $\left({ }^{\circledR}\right)$ anterior segment optical coherence tomography. Acta Ophthalmol. 2014;92(2):e119-123. doi:10.1111/aos.12241
14. Smedt DS, Mermoud A, Schnyder C. 24-hour intraocular pressure fluctuation monitoring using an ocular telemetry sensor: tolerability and functionality in healthy subjects. $J$ Glaucoma. 2012;21 (8):539-544. doi:10.1097/IJG.0b013e31821dac43

15. Agnifili L, Mastropasqua R, Frezzotti P, et al. Circadian intraocular pressure patterns in healthy subjects, primary open angle and normal tension glaucoma patients with a contact lens sensor. Acta Ophthalmol. 2015;93(1):e14-21. doi:10.1111/aos.12408

16. Mansouri K. The road ahead to continuous 24-hour intraocular pressure monitoring in glaucoma. J Ophthalmic Vis Res. 2014;9 (2):260-268.

17. Mountford J. History and general principles. In: Mountford J, Ruston D, Dave T, editors. Orthokeratology. Principles and Practice. Oxford, UK: Butterworth-Heinemann; 2004:1-13.

18. Shinmei Y, Nitta T, Saito H, et al. Continuous intraocular pressure monitoring during nocturnal sleep in patients with obstructive sleep apnea syndrome. Invest Ophthalmol Vis Sci. 2016;57(6):2824-2830. doi:10.1167/iovs.16-19220

19. Morales-Fernandez L, Garcia-Bella J, Martinez-de-la-Casa JM, et al. Changes in corneal biomechanical properties after 24 hours of continuous intraocular pressure monitoring using a contact lens sensor. Can J Ophthalmol. 2018;53(3):236-241. doi:10.1016/j.jcjo.2017.10.028

20. Tojo N, Hayashi A. Influence of ocular dimensional change on 24-hour intraocular pressure measurement with contact lens sensor. J Glaucoma. 2019;28(9):808-810. doi:10.1097/IJG.0000000000001318

21. Marando CM, Mansouri K, Kahook MY, Seibold LK. Tolerability and functionality of a wireless 24-hour ocular telemetry sensor in African American Glaucoma Patients. J Glaucoma. 2019;28 (2):119-124. doi:10.1097/IJG.0000000000001141

22. Holló G, Kóthy P, Vargha P. Evaluation of continuous 24-hour intraocular pressure monitoring for assessment of prostaglandin-induced pressure reduction in glaucoma. J Glaucoma. 2014;23(1):e6-12. doi:10.1097/IJG.0b013e31829e5635

23. Hickson-Curran S, Brennan NA, Igarashi Y, Young G. Comparative evaluation of Asian and white ocular topography. Optom Vis Sci. 2014;91(12):1396-2405. doi:10.1097/OPX.0000000000000413

\section{Publish your work in this journal}

The International Medical Case Reports Journal is an international, peer-reviewed open-access journal publishing original case reports from all medical specialties. Previously unpublished medical posters are also accepted relating to any area of clinical or preclinical science. Submissions should not normally exceed 2,000 words or 4 published pages including figures, diagrams and references. The manuscript management system is completely online and includes a very quick and fair peer-review system, which is all easy to use. Visit http://www.dovepress.com/testimonials.php to read real quotes from published authors. 